\title{
Pareceristas do volume 34
}

Adriana Gomes Dickman - Pontifícia Universidade Católica, Belo Horizonte, MG

Adriano José Ortiz - Instituto Federal do Paraná, Paranavaí, PR

Alessandra Riposati Arantes - Instituto de Física, Universidade Federal de Uberlândia, MG

Alessandro Damásio Trani Gomes - Departamento de Ciências Naturais, Universidade Federal de São João del-Rei, MG

Alessandro Frederico da Silveira - Universidade Estadual da Paraíba, Campina Grande, PB

Alysson Steimacher - Universidade Federal do Maranhão, Imperatriz, MA

Ana Lúcia Pereira Baccon - Universidade Estadual de Londrina, PR

Ana Paula Bispo da Silva - Centro de Ciências e Tecnologia, Universidade Estadual da Paraíba, PB

Andre Ary Leonel - Departamento de Física, Universidade Federal de Santa Maria, RS

André Koch Torres de Assis - Instituto de Física, Universidade Estadual de Campinas, SP

Antonio Jorge Sena dos Anjos - Departamento de Física, Universidade Estadual de Feira de Santana, BA

Antônio Marcelo Martins Maciel - Universidade Federal de Lavras, PR

Avacir C. Andrello - Departamento de Física, Universidade Estadual de Londrina, PR

Boniek Venceslau da Cruz Silva Universidade Federal do Piauí, Teresina, PI

Breno Arsioli Moura - Centro de Ciências Naturais e Humanas, Universidade Federal do ABC, Santo André, SP

Carlos Alexandre dos Santos Batista - Doutorando no Programa de Pós-Graduação em Educação Científica e Tecnológica, Universidade Federal de Santa Catarina, SC

Carlos Eduardo Laburú - Departamento de Física, Universidade Estadual de Londrina, PR

Carlos Henrique da Silva Santos - Instituto Federal de Educação, Ciência e Tecnologia de São Paulo, Campus Itapetininga, SP

Cecil Chow Robilotta - Instituto de Física, Universidade de São Paulo, SP 
Cibelle Celestino Silva - Instituto de Física, Universidade de São Paulo, São Carlos, SP

Danilo Rodrigues César - Universidade Federal do Triângulo Mineiro, Uberaba, MG

Diego D. Uzêda - Centro Federal de Educação Tecnológica Celso Suckow da Fonseca, Rio de Janeiro, RJ

Dileia Martins - Departamento de Psicologia, Universidade Federal de São Carlos, SP

Diogo Amaral de Magalhães - Instituto Federal Catarinense, Campus São Francisco do Sul, SC

Diogo Soga - Instituto de Física, Universidade de São Paulo, SP

Dirceu da Silva - Departamento de Metodologia do Ensino, Universidade Estadual de Campinas, SP

Ederson Staudt - Universidade Federal do Amapá, Macapá, AP

Edmundo Rodrigues Júnior - Instituto Federal de Educação, Ciência e Tecnologia do Espírito Santo, Unidade Cachoeiro de Itapemirim, ES

Eduardo Luiz Dias Cavalcanti -Instituto de Química, Universidade de Brasília, DF

Eliane Rothmund Bolfe - Instituto Federal Catarinense, Concordia, SC

Elio Ricardo - Faculdade de Educação, Universidade de São Paulo, SP

Esdras Garcia Alves - Instituto Federal Minas Gerais, Campus Congonhas, MG

Esther Pacheco de A. Prado - Instituto de Ciências Matemáticas e de Computação, Universidade de São Paulo, São Carlos, SP

Everton Lüdke - Departamento de Física, Universidade Federal de Santa Maria, RS

Fabio da Purificação de Bastos - Centro de Educação, Universidade Federal de Santa Maria, RS

Fabio de Souza Alves - Instituto Federal de Educação, Ciência e Tecnologia, Universidade Federal do Paraná, Capanema, PR

Fernanda Teresa Moro - Universidade Regional Integrada do Alto Uruguai e das Missões, Campus de Erechim, RS

Fernando Lang da Silveira - Instituto de Física, Universidade Federal do Rio Grande do Sul, RS 
Fernando José Luna - Universidade Estadual do Norte Fluminense Darcy Ribeiro, Campos dos Goytacazes, RJ

Flávia Maria Teixeira Santos - Faculdade de Educação, Universidade Federal do Rio Grande do Sul, RS

Francisco Catelli - Departamento de Física e Química, Universidade de Caxias do Sul, RS

Gabriel Dias de Carvalho Júnior - Departamento de Educação, Universidade Federal de Viçosa, MG

Gabriel Dias de Carvalho Júnior - Departamento de Educação, Universidade Federal de Viçosa, MG

Geraldo Fernandes - Universidade Federal dos Vales do Jequitinhonha e Mucuri, Diamantina, MG

Gilda Noemí Dima - Facultad Ciencias Exactas y Naturales, Universidad Nacional de La Pampa, La Pampa, Argentina

Giorjety L. Dias - Instituto Federal de São Paulo, Campus Boituva, SP

Giselle Watanabe Caramello - Centro de Ciências Naturais e Humanas, Universidade Federal do ABC, Santo André, SP

Giuseppi Gava Camilleti - Departamento de Física, Universidade Federal do Espírito Santo, ES

Glauco Cohen Ferreira Pantoja - Universidade Federal do Oeste do Pará, Santarém, PA

Gustavo Antonio Montenegro Guttmann - Centro Federal de Educação Tecnológica Celso Suckow da Fonseca, Nova Friburgo, RJ

Hebert Roberto Araujo Silva - Colégio Brigadeiro Newton Braga, Rio de Janeiro, RJ

Hector Riveros - Instituto de Física, Universidad Nacional Autónoma de México, México

Helena Libardi - Universidade Federal de Lavras, PR

Hélio Salim de Amorim - Instituto de Física, Universidade Federal do Rio de Janeiro, RJ

Henrique Cesar da Silva - Departamento de Metodologia do Ensino, Universidade Federal de Santa Catarina, SC

Ileana María Rosa Greca - Universidad de Burgos, Espanha

Indianara Lima Silva - Departamento de Física, Universidade Estadual de Feira de Santana, BA 
Isabel Krey Garcia - Centro de Ciências Naturais e Exatas, Universidade Federal de Santa Maria, RS

Italo Gabriel Neide - Centro Universitário Univates, Lajeado, RS

Jair Lúcio Prados Ribeiro - Programa de Pós-Graduação em Ensino de Ciências, Universidade de Brasília, DF

Jandrews Lins Gomes - IFPE, Campus Pesqueira, PE

Janice Pereira Lopes - Instituto de Matemática e Estatística, Universidade Federal de Goiás, Campus Samambaia, Goiânia, GO

João Paulo Martins de Castro Chaib - Universidade Católica de Brasília, DF

Joaquim Anacleto - Departamento de Física, Universidade de Trás-os-Montes e Alto Douro, Vila Real, Portugal

Jonas Cegelka da Silva - Instituto Federal Farroupilha, Campus Santa Rosa, RS

José Cláudio Reis - Universidade do Estado do Rio de Janeiro, RJ

José de Pinho Alves Neto - Instituto Federal de Educação, Ciência e Tecnologia de Santa Catarina, SC

José Martins de Oliveira Jr. - Universidade de Sorocaba, Sorocaba, SP

José Paulo Gircoreano - Instituto Federal de São Paulo, São Paulo, SP

José Roberto da Rocha Bernardo - Faculdade de Educação, Universidade Federal Fluminense, Campus do Gragoatá, Niterói, RJ

José Roberto Tagliati - Departamento de Física, Universidade Federal de Juiz de Fora, MG

Jucelino Cortez - Instituto de Ciências Exatas e Geociências, Universidade de Passo Fundo, RS

Judismar Tadeu Guaitolini Junior - Instituto Federal de Educação, Ciência e Tecnologia do Espírito Santo, Vitoria, ES

Julia Flores Espejo - Departamento de Biología y Química, Universidad Pedagógica Experimental Libertador, Caracas, Venezuela

Juliana Mesquita Hidalgo Ferreira Drummond - Departamento de Física Teórica e Experimental, UFRN, RN

Katemari Diogo da Rosa - Instituto de Física, Universidade Federal da Bahia, BA 
Larissa Moreira Ferreira - Universidade Federal de Santa Catarina, Florianópolis, SC

Leonir Lorenzetti - Departamento de Química e Eduquim, Universidade Federal do Paraná, Curitiba, PR

Lisiane Araujo Pinheiro - Instituto Federal Catarinense, Campus Videira, SC

Lúcia Helena Sasseron - Departamento de Metodologia do Ensino e Educação Comparada, Faculdade de Educação, USP, SP

Luciano Carvalhais Gomes - Departamento de Física, Universidade Estadual de Maringá, PR Luciano Soares Pedroso - Universidade Federal dos Vales do Jequitinhonha e Mucuri, Campus JK, Diamantina, MG

Luiz Antonio Dworakowski - Universidade Federal do Pampa, Bagé, RS

Luiz Pinheiro Cordovil da Silva - Departamento de Engenharia de Energia e Automação Elétrica, Universidade de São Paulo, SP

Márcia Maria Lucchese - Universidade Federal do Pampa, Bagé, RS

Márcio Santos Miranda - Núcleo Interdisciplinar de Pesquisa, Ensino e Consultoria, Campinas, SP

Márcio Sousa Amazonas - Instituto Federal de Educação, Ciência e Tecnologia do Amazonas, Manaus, AM

Marcos A. Araújo Silva - Departamento de Física, Universidade Federal do Ceará, Campus do Pici, Fortaleza, Ce

Marcos Fernandes Sobrinho - Instituto Federal Goiano, Campus Urutaí, GO

Marcos Rincon Voelzke - Programa de Mestrado e Doutorado em Ensino de Ciências e Matemática, Universidade Cruzeiro do Sul, SP

Maria Cecilia Pereira Santarosa - Departamento de Matemática, Universidade Federal de Santa Maria, RS

Maria Inês Martins - Programa de Pós-graduação em Ensino de Ciências e Matemática, PUC, MG

Maria Elenir Nobre Pinho Ribeiro - Universidade Federal do Ceará, Fortaleza, CE

Marília Paixão Linhares - Universidade Estadual do Norte Fluminense, Rio de Janeiro, RJ

Marina Vargas Ferreira - Departamento de Construção Civil, Universidade Federal do Paraná, Curitiba, PR 
Marinez Meneghello Passos - Departamento de Matemática, Universidade Estadual de Londrina, PR

Marta Maximo Pereira - CEFET/RJ, Unidade de Ensino Descentralizada de Nova Iguaçu, RJ

Miguel Ángel Ré - Facultad de Matemática, Astronomía, Física y Computación Universidad Nacional de Córdoba, Córdoba, Argentina

Mikiya Muramatsu - Instituto de Física, Universidade de São Paulo, SP

Nelson Canzian da Silva - Departamento de Física, Universidade Federal de Santa Catarina, $\mathrm{SC}$

Nelson Luiz Reyes Marques - Instituto Federal Sul-rio-grandense, RS

Nildo Loiola Dias - Universidade Federal do Ceará, Campus da UFC, Fortaleza, CE

Norberto Aranha - Universidade de Sorocaba, SP

Osmar Henrique Moura da Silva - Departamento de Física, Universidade Estadual de Londrina, PR

Osvaldo Frota Pessoa Junior - Departamento de Filosofia, Universidade de São Paulo, SP

Ourides Santin Filho -Universidade Estadual de Maringá, PR

Paulo Celso Ferrari - Instituto de Física, Universidade Federal de Goiás, Goiânia, GO

Paulo César Costa de Oliveira - Instituto de Química e Biotecnologia, Universidade Federal de Alagoas, Maceió, AL

Paulo Henrique Guadagnini - Universidade Federal da Fronteira Sul, Campus Chapecó, SC

Paulo José Sena dos Santos - Departamento de Física, Universidade Federal de Santa Catarina, SC

Paulo Sérgio Parreira - Departamento de Física, Universidade Estadual de Londrina, PR

Petrus Alcantara Jr. - Departamento de Física, Universidade Federal do Pará, PA

Rafael Campos - Departamento de Física, Universidade Federal de Santa Catarina, SC

Rafael Gallina Delatorre - Universidade Federal de Santa Catarina, Campus de Joinville, SC

Rafael Silva C. - Facultad de Ciencias Naturales y Exactas, Universidad de Playa Ancha de Ciencias de la Educación, Valparaíso, Chile 
Rafael Vidal Aroca - Departamento de Engenharia Mecânica, Universidade Federal de São Carlos, SP

Renné Medeiros de Araújo - Departamento de Física, Universidade Federal de Santa Catarina, $\mathrm{SC}$

Ricardo Floriano - Faculdade de Ciências Aplicadas, Universidade Estadual de Campinas, SP

Ricardo Karam - Department of Science Education da Universidade de Copenhague, Dinamarca

Roberto Saito - Departamento de Física, Universidade Federal de Santa Catarina, SC

Robson de Sousa Nascimento - Departamento de Solos e Engenharia Rural, Universidade Federal da Paraíba, Areia, PB

Rodrigo Drumond Vieira - Faculdade de Educação, Universidade Federal Fluminense, RJ

Ronaldo Silva Rêgo - Universidade Federal de Alagoas, AL

Roseline Strieder - Instituto de Física, Universidade de Brasília, DF

Rosemar Ayres dos Santos - Universidade Federal da Fronteira Sul, Cerro Largo, RS

Rudinei Luiz Bogo - Universidade Positivo, Curitiba, PR

Saionara Moreira A. das Chagas - Instituto Geremário Dantas, Rio de Janeiro, RJ

Samir Lacerda da Silva - Instituto Federal do Espírito Santo, Coordenação de Física, Campus Vitória, ES

Silvio Luiz Rutz da Silva - Departamento de Física, Universidade Estadual de Ponta Grossa, PR

Stenio Octávio de Oliveira Cardoso - Centro Universitário Uma, Belo Horizonte, MG

Thaís Cyrino de Mello - Universidade Federal de São Paulo, Diadema, SP

Tiago Destéffani Admiral - Instituto Federal de Educação, Ciência e Tecnologia Fluminense, Campos dos Goytacazes, RJ

Tobias Espinosa de Oliveira - Departamento de Física, Universidade Federal do Rio Grande do Sul, Porto Alegre, RS

Ubiratan D’Ambrósio- Instituto de Matemática, Universidade Estadual de Campinas, SP Vania Elisabeth Barlette - Centro Universitário Franciscano, Santa Maria, RS 
Wagner Duarte José - Departamento de Ciências Exatas e Tecnológicas, Universidade de Santa Cruz, BA

Wagner Tenfen - Colegiado de Ciências, Universidade Federal da Fronteira Sul, Realeza, PR

Washington Luiz Raposo - Centro Federal de Educação Tecnológica Celso Suckow da Fonseca, Nova Friburgo, RJ

Wilson Lopes - Departamento de Física, Universidade de Guarulhos, SP 